\title{
Disposable electrochemical immunosensor for cortisol determination in human saliva
}

Satu Kämäräinen ${ }^{\diamond}$, Marianne Mäki ${ }^{\diamond}$, Tiina Tolonen, Giuseppe Palleschi, Vesa Virtanen, Laura Micheli* Adama Marie Sesay*

University of Oulu, Kajaani University Consortium, Kehräämöntie 7, 87400 Kajaani, Finland. Tel. +358405673914

Dipartimento di Scienze e Tecnologie Chimiche, Università di Roma “Tor Vergata", Via della Ricerca Scientifica, 00133 Roma - Italy

\footnotetext{
${ }^{\diamond}$ These two authors have equally contributed to this paper as first author

* These two authors have equally contributed to this paper as corresponding author
} 


\begin{abstract}
Cortisol is a steroidal hormone and an important stress marker. Free serum cortisol concentration has been identified to correlate well with free salivary cortisol. In this present work an electrochemical immunosensor was developed to determine cortisol concentration within the physiological concentration range found in human saliva. The immunosensor is based on a direct competitive enzyme linked immunoassay using a home-made cortisol-alkaline phosphatase (AP) conjugate synthesized in our laboratory with disposable graphite screen-printed electrodes (SPEs). 1-nalphtyl phosphate (1-NP) was used as an enzymatic substrate and a square wave voltammetry (SWV) for electrochemical detection. To study method suitability for use with saliva samples, calibration curves were performed both in buffer and saliva. In buffer standard samples showed a limit of detection (LOD) of $0.6 \mathrm{ng} / \mathrm{ml}$ and working range (WR) of $0.2-44.6 \mathrm{ng} / \mathrm{ml}$ with good reproducibility (RSD 10\%). Saliva matrix effect was removed effectively with Salivette Cortisol collection device (polyethylene) and a calibration curve showed similar characteristics as in buffer with LOD $1.7 \mathrm{ng} / \mathrm{ml}$ and WR $0.5-55.1 \mathrm{ng} / \mathrm{ml}$ (RSD 8\%) demonstrating the possibility to determine human salivary cortisol within the desired human physiological range. Spiked saliva samples were analyzed with the developed immunosensor presenting excellent $92-114 \%$ recovery. Comparison to liquid chromatography tandem mass spectrometry (UHPLC-MS/MS) method showed strong 0.90 correlation between methods indicating good accuracy of the developed immunosensor.
\end{abstract}

Keywords: cortisol; saliva; immunoassay; matrix effect; screen-printed electrode; saliva sampling

\title{
1. Introduction
}

Cortisol (hydrocortisone; 11ß-11,17,21-trihydroxypregn-4-ene-3,20-dione) is a glucocorticoid steroid hormone produced in humans by the adrenal glands. The secretion and circulation of cortisol in blood has a circadian rhythm where its concentration is at its highest early in the morning and lowest during the night (Morineau et al., 1997; Nomura et al, 1997). Cortisol is a known "stress hormone" because its secretion can 
be induced by physical and psychological stress (Pruessner et al., 1999; Gatti et al., 2009; Holsboer et al., 2010). Steroidal hormones have an important function within the endocrine, neuronal and immune systems as stress, shock or inflammation can affect glucocorticoid concentration level in biological fluid. Glucocorticoids steroidal hormones are found in free (unbound) and conjugated (bound) form in serum and urine. In saliva steroidal hormones are only present in its free form (Nozaki 2001), due to diffusion through the cells of salivary glands (Vining et al., 1983). It has been demonstrated that salivary cortisol concentration level correlates well with free cortisol concentration in plasma (Vining et al., 1983; Gozansky et al., 2005; Arafah et al., 2007).

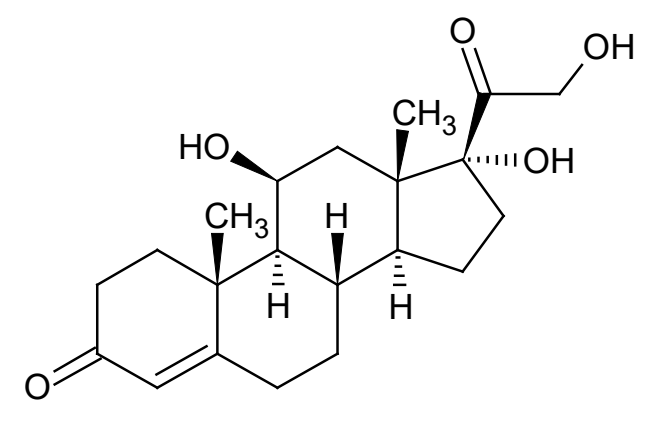

Figure 1. Structure of cortisol

Non-invasiveness and ease of sampling compared to blood makes saliva an attractive biological matrix of choice for analytical purposes related to personal point of care (POC) monitoring for sport research, coaching and well-being services. Saliva sampling offers a stress-free sampling method especially suitable for elderly people and children. In clinical applications salivary cortisol level could also be used to screen for Cushing's syndrome in children (Gafni et al., 2000), hypocortisolism in chronic fatigue syndrome patients (Strickland et al., 1998) and depressive disorders as stress is associated with increased cortisol concentration (Pruessner et al., 1999). Also correlation between anxiety and depressive symptoms with increased salivary cortisol concentration has been observed in studies with rescue workers (Aardal-Eriksson et al., 1999). 
Saliva is a complex biological fluid matrix which composition can vary inter-individually and intraindividually (Lewis, 2006; de Almeida et al., 2008). Saliva is produced mainly by the parotid, submandibular and sublingual salivary glands, with a small proportion also formed in buccal glands. There are several reported ways in which saliva samples can be collected for cortisol determination. Sarstedt's Salivette saliva collection devices have been used in many studies (Jönsson et al., 2003; Gröschl et al., 2006; Gröschl et al., 2008; Perogamvros et al., 2010; Miller et al., 2013) and other devices such as Quantisal (Immunalysis) and SCS (Greiner Bio-One) (Gröschl et al., 2008) are also commercially available. Likewise passively drooled (Anfossi, et al., 2002; Yamaguchi et al., 2013) or spitted saliva (Gafni et al., 2000) are generally used for saliva sampling. Saliva collection devices have the advantage over needles for blood in that they are non-invasive, more user-friendly and might also reduce matrix problems by removing interfering compounds and molecules.

Many different analytical techniques have been used for quantifying cortisol from human saliva e.g liquidchromatography (Jönsson et al., 2003; Perogamvros et al., 2009) and also immunochemical assays are commonly used (Anfossi et al., 2002; Sesay et al., 2013; Yamaguchi et al., 2013). Liquid chromatography, especially with tandem mass spectrometry detection system, is a very sensitive method and it is not affected by the presence of other steroids in the sample. However, the laboratory instrument is expensive and the saliva needs to be pretreated before analysis. Advantage of immunoassay methods are that they can be equally as sensitive and can easily lend themselves to be turn into portable, hand-held devices, which can be used in field conditions (Kaushik et al., 2014). Ultrasensitive biosensors that measure cortisol in buffer (Arya et al., 2011; Kumar et al., 2007; Vasudev et al., 2013), interstitial fluid (Arya et al., 2010; Venogupal et al., 2011) or artificial saliva (Tlili et al., 2011) has been developed showing high sensitivity that can be reached with immunoassay approach.

Real saliva sample exhibit strong matrix effect and to avoid this different techniques and methods have been used when analyzing steroids from human saliva (Anfossi et al., 2002; Mitchell et al., 2009; Sesay et al., 2013). Immunosensors for salivary cortisol measurement have been developed by Arya et al. (2010), 
Sun et al. (2008) and Yamaguchi et al. (2013). Arya et al. (2010) successfully removed saliva matrix effect by diluting samples $100 \mathrm{x}$ with PBS buffer and reached 1 pM-100 nM linear range. Sun et al. (2008) quantified cortisol from whole saliva with good detection limit using cyclic voltammetry and reached 0.3 $\mathrm{ng} / \mathrm{ml}$ detection limit whereas Yamaguchi et al. (2013) used fluid control system with electrochemical measurement and achieved $0.1-10 \mathrm{ng} / \mathrm{ml}$ linear range.

The aim of this work was to develop a disposable immunosensor for human salivary cortisol determination based on a house-made cortisol-alkaline phosphatase (AP) conjugate and screen-printed electrodes (SPEs), which are easy to use and inexpensive. We studied saliva matrix effect comprehensively and tested two commercially available saliva collection devices and their suitability for cortisol sampling. The determination of cortisol in saliva has been carried out using a direct competitive ELISA format with electrochemical detection. All analytical parameters, such as concentrations of the reagents, electrochemical method and parameters were studied and optimized in order to obtain high reproducibility, repeatability and sensitivity of the analysis. Immunosensor results were compared with ultra-high pressure liquid chromatography tandem mass spectrometry (UHPLC-MS/MS).

\section{Material and methods}

\subsection{Chemicals and bioreagents}

Affinity chromatography purified goat anti-mouse IgG H/L (approx. $2.0 \mathrm{mg} / \mathrm{ml}$; $\mathrm{Ab}$ II) was from $\mathrm{AbD}$ Serotec (Kidlington, UK). Exchange chromatography purified monoclonal mouse anti-cortisol (mAb) was from HyTest Ltd (Turku, Finland). Hydrocortisone 3-carboxymethyloxime (CS-oxime) and alkaline phosphatase (AP; from bovine intestinal mucosa, EC 3.1.3.1) were from Sigma-Aldrich (Darmstadt, Germany). Hydrocortisone-D4 (internal standard for UHPLC-MS/MS) was from Cambridge Isotope Laboratories Inc. (Tewksbury, USA). $N$-hydroxysulfosuccinimide sodium salt (sulfo-NHS) and (2hydroxypropyl)- $\beta$-cyclodextrin $\quad$ (HP $\beta C D) \quad$ were $\quad$ from $\quad$ Aldrich $\quad$ (Sigma-Aldrich). $N$-(3- 
dimethylaminopropyl)- $N$ '-ethylcarbodiimide hydrochloride (EDC), hydrocortisone (cortisol), hydrogen peroxide $\left(\mathrm{H}_{2} \mathrm{O}_{2}\right)$ and methanol (LC-MS grade) were from Fluka (Sigma-Aldrich). 1-naphthyl phosphate disodium salt (1-NP), polyvinyl alcohol (PVA), phosphate buffered saline (10 mM PBS: 3 mM KCl, 0.137 M NaCl, pH 7.4), PBS containing Tween 20 (0.05\% Tween 20, pH 7.4), 4-Morpholineethanesulfonic acid hemisodium salt (MES) and ascorbic acid (AA) were from Sigma (Sigma-Aldrich). Copper(II) sulfate pentahydrate $\left(\mathrm{CuSO}_{4} * 5 \mathrm{H}_{2} \mathrm{O}\right)$, disodium hydrogen phosphate $\left(\mathrm{Na}_{2} \mathrm{HPO}_{4}\right)$, citric acid monohydrate, ethyl acetate and water (LC-MS grade) were products of (VWR) Merck (Darmstadt, Germany). AP-StabilPLUS was from Kem-En-Tec Diagnostics A/S (Taastrup, Denmark), Tween 20 was from J.T. Baker (OneMed Oy, Helsinki, Finland) and diethanolamine (DEA) buffer solution (Alkaline Phosphatase Substrate Kit, BioRad Laboratories, Hercules, CA, USA). All reagents were of analytical grade unless otherwise stated.

\subsection{Buffer solutions}

$50 \mathrm{mM}$ carbonate buffer $\mathrm{pH} 9.6\left(\mathrm{CB}: 15 \mathrm{mM} \mathrm{Na}_{2} \mathrm{CO}_{3}, 35 \mathrm{mM} \mathrm{NaHCO}_{3}\right)$ was used for the immobilization of anti-mouse $\mathrm{Ab}_{\mathrm{II}}$ on the electrode surface. $10 \mathrm{mM}$ PBS $\mathrm{pH} 7.4$ buffer was used for the addition of the primary antibody $(\mathrm{mAb})$ on the working electrode and also in washing steps. $1 \%(\mathrm{w} / \mathrm{v}) \mathrm{HP} \beta \mathrm{CD}$ in $50 \mathrm{mM}$ Tris-buffered saline (TBS: $50 \mathrm{mM}$ Tris-HCl, $150 \mathrm{mM} \mathrm{NaCl}, \mathrm{pH}$ 7.4) was used for the competition step and prepared daily. PBS-Tween and TBS with added $0.05 \%(\mathrm{w} / \mathrm{v})$ Tween 20 (TBS-Tween) were used as washing solutions. Citrate-phosphate buffer $\mathrm{pH} 5.7$ (CP: $0.2 \mathrm{M}$ phosphate, $0.1 \mathrm{M}$ citrate) was used for saliva dilution in matrix effect study. Daily prepared 0.97 M DEA buffer (pH 9.8) with $0.08 \mathrm{M} \mathrm{KCl}$ and $1.0 \mathrm{mM}$ $\mathrm{MgCl}_{2}$ was used as enzymatic substrate and prepared daily. Ethanol (Rajamäki, Finland) was used to dissolve CS-oxime in preparation of cortisol-AP conjugate and also to dissolve cortisol into $1 \mathrm{mg} / \mathrm{ml}$ stock solution.

\subsection{Equipment and commercial analytical kits}


The electrochemical measurements were performed using an EmStat potentiostat (PalmSens BV, Netherlands) equipped with PSTrace software. Disposable SPEs were obtained from EcoBioServices \& Researches (Florence, Italy). SPEs consisted of a graphite working electrode (WE; $3 \mathrm{~mm} \varnothing)$ with an $\mathrm{Ag} / \mathrm{AgCl}$ reference electrode and a graphite counter electrode.

Commercial salivary cortisol enzyme immunoassay kits were purchased from Salimetrics Europe Ltd (Suffolk, UK). Salivette and Salivette Cortisol saliva collection tubes were from Sarstedt AG \& Co. (Nümbrecht Germany). Microcon YM-10 centrifugal filter devices were from Millipore Corp (Billerica, MA, USA). BCA Protein Assay Kit was from Pierce (VWR International Oy, Finland) and Varioskan Flash plate reader was from Thermo Fisher Scientific (Vantaa, Finland).

\subsection{Cortisol-alkaline phosphatase conjugate preparation}

The cortisol-AP conjugate was prepared using a modified method developed in our laboratory (Sesay et al., 2013). The cortisol-AP was prepared by adding $2.2 \mathrm{mg}$ CS-oxime in $250 \mu 1$ ethanol and $250 \mu 1 \mathrm{MES} \mathrm{pH}$ 6.0 and mixing with a vortex mixer until CS-oxime was totally dissolved into solution. $2.2 \mathrm{mg}$ sulfo-NHS in $250 \mu 1 \mathrm{MES}$ and $9.6 \mathrm{mg}$ EDC in $250 \mu 1 \mathrm{MES}$ were added to the CS-oxime solution and left to react at room temperature (RT) on block shaker for one hour. After shaking $175 \mu 1$ of CS-oxime:sulfo-NHS:EDC -solution was mixed with $700 \mu$ of AP $(12.5 \mu \mathrm{M}$, diluted into PBS buffer) and left to react on a block shaker at $+4{ }^{\circ} \mathrm{C}$ overnight. Resulting cortisol-AP conjugate was purified with Microcon YM-10 centrifugal filter device. After purification, the protein concentration of cortisol-AP was determined spectrophotometrically at $595 \mathrm{~nm}$ using BCA Protein Assay Kit according to the kit instructions giving a protein concentration of $4.2 \mathrm{mg} / \mathrm{ml}$. The conjugate solution was diluted with AP-StabilPLUS to the concentration $1 \mathrm{mg} / \mathrm{ml}$. The diluted conjugate was stored at $+4{ }^{\circ} \mathrm{C}$ until required.

\subsection{Optimization of electrochemical detection method}


Alkaline phosphatase is a hydrolase enzyme that catalyzes 1-NP hydrolysis to 1-naphthol in a one electron transfer reaction that can be detected electrochemically (Preechworapun et al., 2008). The 1-NP itself is not electroactive so all electrochemical response comes from enzymatic product 1-naphthol which oxidation reaction is irreversible. Electroactive behavior of 1-NP was studied first using cyclic voltammetry $(\mathrm{CV})$ and the optimal potential range for oxidation was found to be $0.05-0.5 \mathrm{~V}$ similarly as found in literature (Gehring et al. 1999). By using this range other parameters (step and amplitude potential, frequency) for square wave voltammetry (SWV) were optimized. Experiments showed that by increasing amplitude and frequency, the signal intensity increased but also background of immunoassay and standard deviation (SD) increased. Therefore $25 \mathrm{mV}$ amplitude and $25 \mathrm{~Hz}$ frequency was chosen as they gave high response with low background and small SD values. Step potential of $4 \mathrm{mV}$ is used in multitude of experiments with similar amplitude and frequency values (Gehring et al., 1999; Liu et al., 2006; Lee et al., 2008).

\subsection{Optimized immunosensor protocol}

Schematic representation of direct competitive ELISA method, built on the working electrode of SPE and proposed in this paper, is presented in figure 2. SPEs were washed with PBS (pH 7.4) and dried after which WE was precoated with $6 \mu$ of secondary antibody $\mathrm{Ab}_{\mathrm{II}}(20 \mu \mathrm{g} / \mathrm{ml}$ in $\mathrm{CB}, \mathrm{pH} 9.6)$ and incubated $+8{ }^{\circ} \mathrm{C}$ overnight at $40 \%$ relative humidity. To block the residue free surface of WE, $6 \mu 1$ of $0.5 \%(\mathrm{w} / \mathrm{v})$ PVA in $\mathrm{CB}(\mathrm{pH}$ 9.6) was applied to the WE surface and left for 30 minutes at RT on block shaker set at a gentle

rotation speed. For the coating, $6 \mu 1$ of primary antibody mAb $(2.5 \mu \mathrm{g} / \mathrm{ml}$ in PBS, $\mathrm{pH} 7.4)$ was applied to the WE and left to immobilize for $60 \mathrm{~min}$ on the block shaker at RT. For the competition step, equal volumes of cortisol-AP (1:6000 v/v dilution) and cortisol standard (0-400 ng/ml) in buffer or in saliva were mixed together and then $6 \mu 1$ of the mixture was placed on the WE. The competition reaction was left to proceed at RT on a block shaker for 30 minutes. After each step electrodes were washed twice with $100 \mu$ PBS- 
Tween and twice with $100 \mu \mathrm{l}$ PBS, except after competition when TBS-Tween and TBS were used instead. After each washing step, SPEs were left RT to dry before adding new reagents to the WE.

The electrochemical response was measured after adding $50 \mu 1$ of $1-\mathrm{NP}(1.5 \mathrm{mg} / \mathrm{ml})$ in DEA solution to the SPE and incubating 2 minutes. SWV was performed over a potential range of $0.05-0.5 \mathrm{~V}$ with a step potential of $4 \mathrm{mV}$ using $25 \mathrm{mV}$ amplitude and $25 \mathrm{~Hz}$ frequency. A blank control was always included.

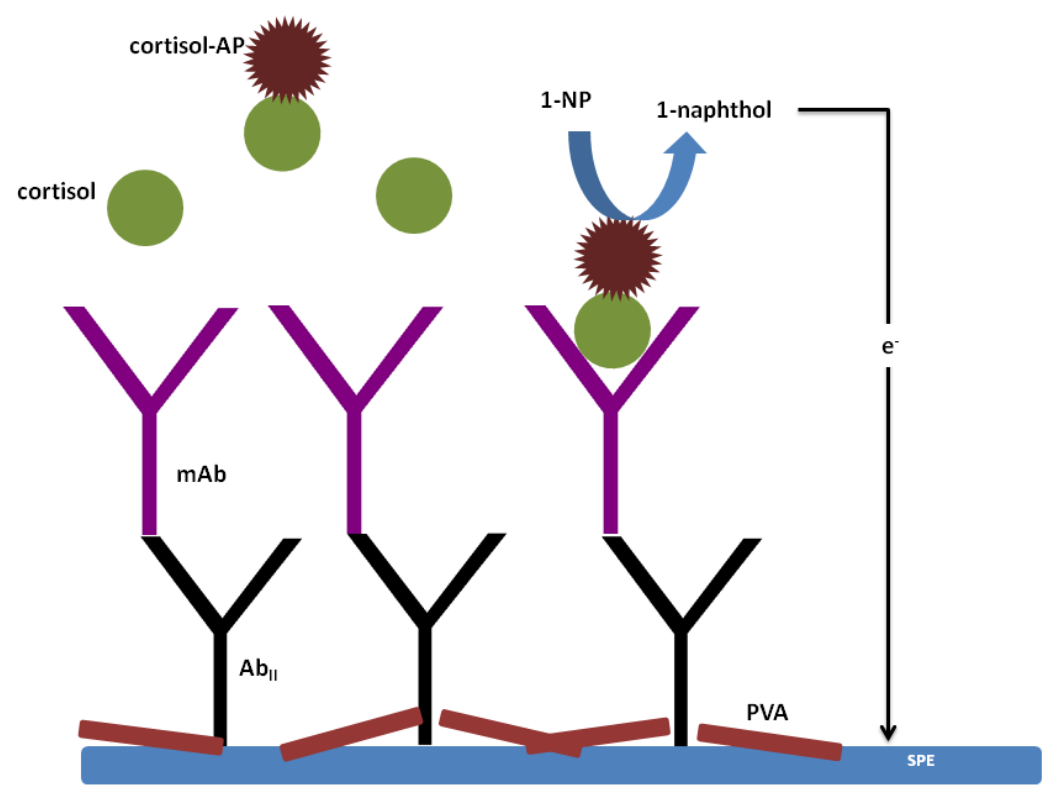

Figure 2. Schematic diagram of the immunoassay procedure. WE of SPE was precoated with $A b_{\text {II }}$ and the residue surface was blocked with PVA. mAb was applied to WE and after that competition step with cortisol-AP and cortisol (standard/sample) was left to proceed. After adding 1-NP, electrochemical response was measured with SWV.

\subsection{UHPLC-MS/MS protocol}

Analysis were conducted using Shimadzu UHPLC liquid chromatograph Nexera $\mathrm{X}^{2}$ coupled to Shimadzu triple quadrupole mass spectrometer 8050 (Shimadzu, Kyoto, Japan) equipped with ESI ion source. Samples were cooled to $15^{\circ} \mathrm{C}$ before injection of $25 \mu 1$ in the autosampler. Kinetex EVO C18 column (50 
x $2.1 \mathrm{~mm}, 2.6 \mu \mathrm{m}$; Phenomenex) was used for separation in temperature of $40{ }^{\circ} \mathrm{C}$ in conjunction with water as mobile phase A and methanol as B. The gradient modified from Phenomenex Steroids Panel application (application ID 20655) was as follows: 0-2 $\min , 90 \% \mathrm{~A} ; 2-3 \mathrm{~min}, 90-60 \% \mathrm{~A} ; 3-7 \mathrm{~min}, 60-30 \% \mathrm{~A}$; 7-7.5 $\min , 30-5 \% \mathrm{~A} ; 7.5-10.5 \mathrm{~min}, 5 \% \mathrm{~A} ; 10.5-10.7 \min 5-90 \%$, A; 10.7-12.5 $\mathrm{min}, 90 \%$, and flow rate 0.3 $\mathrm{ml} / \mathrm{min}$.

Stock solutions of cortisol standard and internal standard were prepared to methanol. For quantification, seven dilutions between $0.1-25 \mathrm{ng} / \mathrm{ml}$ of hydrocortisone in $10 \%$ methanol were made. Quantitation was performed with positive ion mode at interface temperature of $300{ }^{\circ} \mathrm{C}$, heating block temperature of $400{ }^{\circ} \mathrm{C}$ and desolvation line temperature of $150^{\circ} \mathrm{C}$, using nebulizer gas flow of $3 \mathrm{ml} / \mathrm{min}$, heating gas flow of 10 $\mathrm{ml} / \mathrm{min}$ and drying gas flow of $10 \mathrm{ml} / \mathrm{min}$. Interface voltage was $4 \mathrm{kV}$. For quantification multiple reaction monitoring (MRM) was used with individual precursor and product ions optimized as: $\mathrm{m} / \mathrm{z} 363.0$-> m/z 121.1 for hydrocortisone and m/z 367.0 -> m/z 121.1 for hydrocortisone-D4. The collision energy was -22 $\mathrm{eV}$ for both compounds.

\subsection{Saliva sampling}

Saliva was collected using the passive drooling method and with two different commercial collection devices: original Salivette (cotton swab) and Salivette Cortisol (polyethylene swab). Eating and drinking was not allowed for 30 minutes before saliva sample collection. Salivette or a Salivette Cortisol swab was placed into the mouth for 2-3 minutes to allow the swab to be totally moistened after which swab was placed in to a collection tube and centrifuged (1000 g, $2 \mathrm{~min})$. Centrifuged saliva samples were pooled and stored in a freezer at $-20{ }^{\circ} \mathrm{C}$ if not assayed on the same day. The saliva samples endogenous cortisol level were determined by using a commercial enzyme immunoassay kit (Salimetrics). 
Electrochemical behavior of saliva collected by each of saliva sampling technique was investigated using CV method.

For the matrix effect studies it was important to use the same saliva sample pool for each of different pretreatments and therefore saliva was collected by passive drool. $1 \mathrm{ml}$ of saliva was used in each pretreatments which were syringe filtration (regenerated cellulose), dilution (TBS with $1 \%(\mathrm{w} / \mathrm{v}) \mathrm{HP} \beta \mathrm{CD}$ $\mathrm{pH} 7.4$ or $\mathrm{CP}$ buffer $\mathrm{pH}$ 5.7), addition of ascorbic acid (AA) and $\mathrm{CuSO}_{4}$ or $\mathrm{AA}$ and hydrogen peroxide $\left(\mathrm{H}_{2} \mathrm{O}_{2}\right)$ in PBS. When Salivette or Salivette Cortisol was used, saliva was adsorbed in swab that was then centrifuged and recovered saliva collected.

For UHPLC-MS/MS analysis saliva samples were collected with polyethylene Salivette Cortisol swabs. Swabs were centrifuged and pooled as described above. Samples were spiked with cortisol and stored in freezer $\left(-20^{\circ} \mathrm{C}\right)$ until analyzed. At the day of analysis, samples were extracted with modified liquid-liquid extraction (LLE) by Jensen et al. (2011). Frozen saliva samples were thawed in RT for 1 hour, mixed and centrifuged with $3500 \mathrm{~g}$ for $10 \mathrm{~min}$ at RT. Hydrocortisone-D4 (in concentration of $5 \mathrm{ng} / \mathrm{ml}$ in $10 \%$ methanol) of $40 \mu 1$ was added to $250 \mu 1$ of supernatant as an internal standard. LLE was carried out with 1 $\mathrm{ml}$ of ethyl acetate by shaking mixture for $45 \mathrm{~min}$ in $\mathrm{RT}$, centrifuging for $5 \mathrm{~min}$ at $3500 \mathrm{~g}$ in $\mathrm{RT}$ and placing tubes in to the freezer for $40 \mathrm{~min}$. Upper ethyl acetate layer was pipetted into a new tube and evaporated to dryness under a steam of nitrogen. Residue was dissolved to $200 \mu 110 \%$ methanol and analyzed in UHPLCMS/MS.

\subsection{Data analysis}

The electrochemical immunoassay data was used to plot standardized calibration curves and were fitted using a nonlinear four parameter logistic (4PL) curve (Rodbard 1974, Krotzky \& Zeeh 1995). The four parameter logistic function is:

$$
y=\frac{(a-d)}{1+\left(\frac{x}{c}\right)^{b}}+d
$$


where $y$ is the response obtained, parameters $a$ and $d$ are asymptotic maximum and minimum of the sigmoidal curve, respectively. Parameter $c$ is the concentration at the inflection point $\left(\mathrm{IC}_{50}\right), b$ is the hillslope and $x$ is the concentration of analyte of interest.

In order to compare calibration curves directly each data points were converted into their corresponding competition values $\left(\% \mathrm{I} / \mathrm{I}_{0}\right)$ as follows:

$$
\% \frac{I}{I_{0}}=100 \times \frac{I-I_{\text {sat }}}{I_{0}-I_{\text {sat }}}
$$

where $I$ is the peak current of the competitor, $I_{\text {sat }}$ is the peak current corresponding to the saturating analyte and $I_{0}$ corresponds to the non-competition analyte (Krotzky \& Zeeh 1995).

The limit of detection (LOD) was defined as the analyte concentration where decrease of the maximum response equals three times the value of SD measured in absence of the analyte $\left(\mathrm{I}_{0}-3 \mathrm{xSD}\right)(\mathrm{McNaught} \&$ Wilkinson 2014, Long \& Winefordner 1983). The working range (WR) was determined as the cortisol concentration that gives competition values of 90 and $10 \%$ of $\% \mathrm{I} / \mathrm{I}_{0}$. Within the WR the slope of the regression line between response and concentration defines the sensitivity (S) of the analytical calibration curve to the given analyte.

\section{Results and discussion}

\subsection{Optimization of electrochemical immunoassay}

The aim of this work was to develop an electrochemical cortisol immunosensor sensitive enough to monitor physiological cortisol concentrations in saliva which are around $0.7-13 \mathrm{ng} / \mathrm{ml}$ (Morineau et al., 1997; Anfossi et al., 2002). SWV method was optimized for this work as well as reagent concentrations for every step (pre-coating, coating, competition and detection) were independently optimized. 
Electrochemical behavior of saliva was investigated to verify that molecules within saliva did not interfere with the determination of cortisol. Therefore saliva was sampled using different collection methods and its electrochemical activity was determined using CV method. No electrochemical activity in saliva was observed within the CV potential range used in this work indicating the absence of electrochemically active compounds in saliva (figure 3).

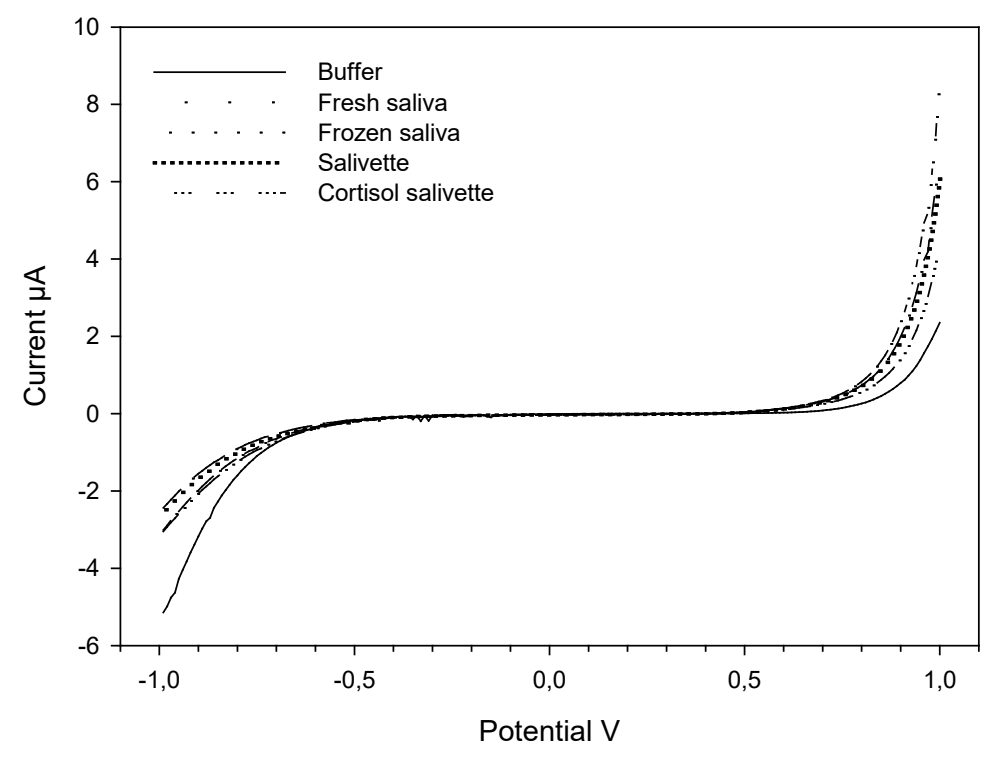

Figure 3. Electrochemical activity in PBS buffer, fresh or frozen saliva collected by passive drool, or in saliva collected with Salivette or Salivette Cortisol. CV settings: range -1 to $1 \mathrm{~V}$, step $10 \mathrm{mV}$ and rate $50 \mathrm{mV} / \mathrm{s}$.

The first optimization step was to optimize the $A b_{\text {II }}$ concentration immobilized on WE. The mouse $A b_{\text {II }}$ orientates the $\mathrm{mAb}$ in an upright direction to afford better binding to the cortisol within the sample. This improved binding orientation increases the response level and provides the sensitivity needed for analyte detection at low concentration. Therefore optimization of the binding surface was important to achieve uniform layers of $A b_{\text {II }}$ and to avoid the formation of multi layers of $A b_{\text {II }}$ and steric hindrance on the WE.

$\mathrm{Ab}_{\text {II }}$ concentrations of $3.8-60.0 \mu \mathrm{g} / \mathrm{ml}$ were absorbed on WE and concentration $20 \mu \mathrm{g} / \mathrm{ml}$ was chosen for further work as a sufficient response level was reached without the electrode surface being saturated (figure 
4a). Higher concentrations would result even higher response but also the risk of steric hindrance increases (intra-experiment RSD $21 \%, n=5$ ). With the optimized $\mathrm{Ab}_{\mathrm{II}}$ concentration different amounts of anticortisol mAb was used in a concentration range of $0.12-8.0 \mu \mathrm{g} / \mathrm{ml}$ (figure $4 \mathrm{~b}$ ) for the coating study. The anti-cortisol mAb concentration of $2.5 \mu \mathrm{g} / \mathrm{ml}$ was selected as it gave a good response without being saturated on the surface (intra experiment RSD $5 \%, n=7$ ).

Cortisol-AP binding study was performed using several conjugate dilutions from 1:16000 to 1:125 (v/v) and a dilution 1:12000 (v/v) of cortisol-AP conjugate was selected as it gave a high response while still within the linear section of the response curve (figure 4c, intra experiment RSD $13 \%, n=7$ ). In the direct competitive immunoassay, the competition binding highly affects the sensitivity of the method. In the competition binding reaction the unbound analyte (sample or standard) and enzyme conjugated analyte competes for the binding sites of the immobilized antibodies on the WE. Changing the conjugate concentration affects the sensitivity of the method which is improved with lower reagent concentrations. The chosen concentration is a compromise between the required sensitivity and signal intensity. When the conjugate concentration is low there is reduced steric hindrance between molecules and also more competition between low concentrations of unbound analyte and conjugate (Pemberton et al., 2005).

\subsection{Optimization of substrate concentration}

The amount of substrate used in measurement step was also optimized because substrate concentration affects electrochemical response intensity. In an ideal situation all bound enzyme conjugates are used in catalyzing the substrate molecules to get the maximal amount of enzymatic product and thus maximal electrochemical response (Rao et al., 2005; Lin et al., 2008). The substrate 1-NP was studied in concentrations $0.5,1.0,1.5$ and $2.0 \mathrm{mg} / \mathrm{ml}$ to achieve the maximal response where all bound cortisol-AP is

involved in the enzymatic reaction. The substrate gave the highest response at $1.5 \mathrm{mg} / \mathrm{ml}$ concentration and 
further increment did not improve response. Therefore $1.5 \mathrm{mg} / \mathrm{ml} 1-\mathrm{NP}$ concentration was chosen for further experiments (see figure $4 \mathrm{~d}$ ).
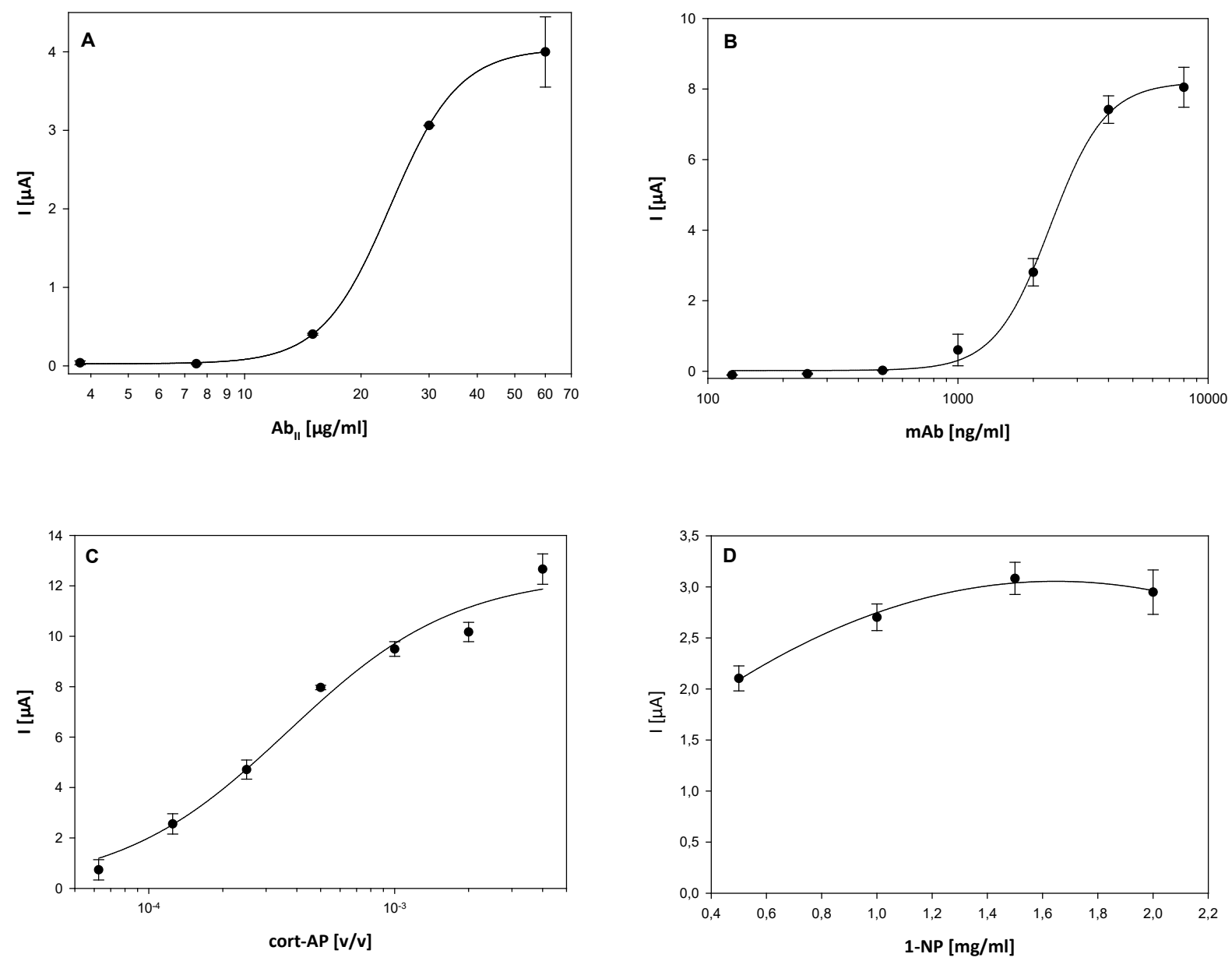

Figure 4. Optimization of electrochemical immunoassay procedure: A) Pre-coating for $\mathrm{Ab}_{\mathrm{II}}, \mathbf{B}$ ) Coating for $\left.\mathrm{mAb}, \mathbf{C}\right)$ Binding for cortisol-AP and D) Substrate (1-NP) concentration studies. SWV settings: $0.05-0.5 \mathrm{~V}$ range, $4 \mathrm{mV}$ step potential, $25 \mathrm{mV}$ amplitude and $25 \mathrm{~Hz}$ frequency.

\subsection{Saliva matrix effect studies}

Saliva is a complex biological fluid where large proteins such like mucin can interfere with the immunoassay and contribute to what is known as the matrix effect of saliva. Several saliva pretreatments were studied to reduce and/or remove matrix interference. Saliva samples collected by passive drool were 
filtered or diluted with different buffers in attempts to remove interfering compounds and also saliva collection devices were tested.

Saliva pretreatments included syringe filtering of saliva with regenerated cellulose membrane, diluting saliva with TBS with $1 \%(\mathrm{w} / \mathrm{v}) \mathrm{HP} \beta \mathrm{CD}(\mathrm{pH}$ 7.4) or CP buffer (pH 5.7), adding ascorbic acid (AA) and $\mathrm{CuSO}_{4}$ or AA and hydrogen peroxide $\left(\mathrm{H}_{2} \mathrm{O}_{2}\right)$ in PBS to saliva sample. AA and $\mathrm{H}_{2} \mathrm{O}_{2}$ have been shown to degrade mucin (Robertson et al., 1941; Pillai et al., 2012) and diluting saliva into acidic buffer has also been reported to reduce the matrix effect of saliva (Anfossi et al., 2002). Also Salivette (cotton) and Salivette Cortisol (polyethylene) swabs were included in study as commercially available sampling devices. All samples were measured using developed immunosensor protocol and responses of pretreated samples were compared to response of the standard solution sample. Standard solution was prepared by adding the same amount of cortisol to buffer ( $1 \% \mathrm{w} / \mathrm{v}$ HP $\beta C D$ in TBS) that was determined from tested saliva. The concentration of endogenous cortisol in the saliva sample was predetermined using the salivary cortisol enzyme immunoassay kit.

Without or with any studied pretreatment, most saliva samples gave higher electrochemical responses than the standard solution with the same amount of cortisol (table 1). This implies that all cortisol in samples is not reacting with $\mathrm{mAb}$ for some reason. For example cortisol might not be recovered from regenerated cellulose filter and diluting with buffers were not an effective mean to reduce the matrix interferences and cortisol is trapped in saliva matrix. Adding AA either with $\mathrm{CuSO}_{4}$ or with $\mathrm{H}_{2} \mathrm{O}_{2}$ increases electrochemical response even higher and this is likely due to the electrochemical activity of ascorbic acid. The highest response was from saliva which was absorbed using a Salivette cotton swab (table 1). This suggests that cortisol was not recovered completely from cotton swab. Gröschl et al. (2008) reported results where cortisol recovery has been $81 \pm 2 \%$ from cotton swab (Salivette) and $98 \pm 4 \%$ from polyethylene swab (Salivette Cortisol) and Strazdins et al. (2005) reported $64 \%$ recovery from Salivette cotton swab. 
Also in this study the Salivette Cortisol polyethylene swab showed high recovery and the same level of response (102\%) was obtained as was in standard solution (table 1). Therefore Salivette Cortisol proved to be the most efficient in removing the interfering compounds in saliva compared to other pretreatments studied in this paper.

Table 1. Effect of saliva pretreatments on electrochemical maximum response (I).

\begin{tabular}{lcc}
\hline Pretreatment & $\mathbf{I}_{\text {sample }} / \mathbf{I}_{\text {std }} \mathbf{( \% )}$ & RSD\% \\
\hline cortisol standard solution & 100 & 3.6 \\
untreated saliva & 111 & 12.6 \\
filtered saliva & 116 & 1.0 \\
TBS diluted saliva & 113 & 1.8 \\
$\mathrm{CP}$ diluted saliva & 109 & 4.4 \\
addition of AA in $\mathrm{CuSO}_{4}$ & 138 & 3.8 \\
addition of AA in $\mathrm{H}_{2} \mathrm{O}_{2}$ & 121 & 1.9 \\
Salivette cotton swab & 157 & 1.3 \\
Salivette Cortisol polyethylene swab & 102 & 7.5 \\
\hline
\end{tabular}

Using the optimized electrochemical immunoassay protocol, calibration curves were performed in buffer and saliva collected with Salivette cortisol (figure 5). The calibration curve performed in buffer gave a LOD of $0.6 \mathrm{ng} / \mathrm{ml}$ and WR was $0.2-44.6 \mathrm{ng} / \mathrm{ml}$ and showed good reproducibility (RSD 10\%). With this working range it is possible to determine physiological salivary cortisol concentrations.

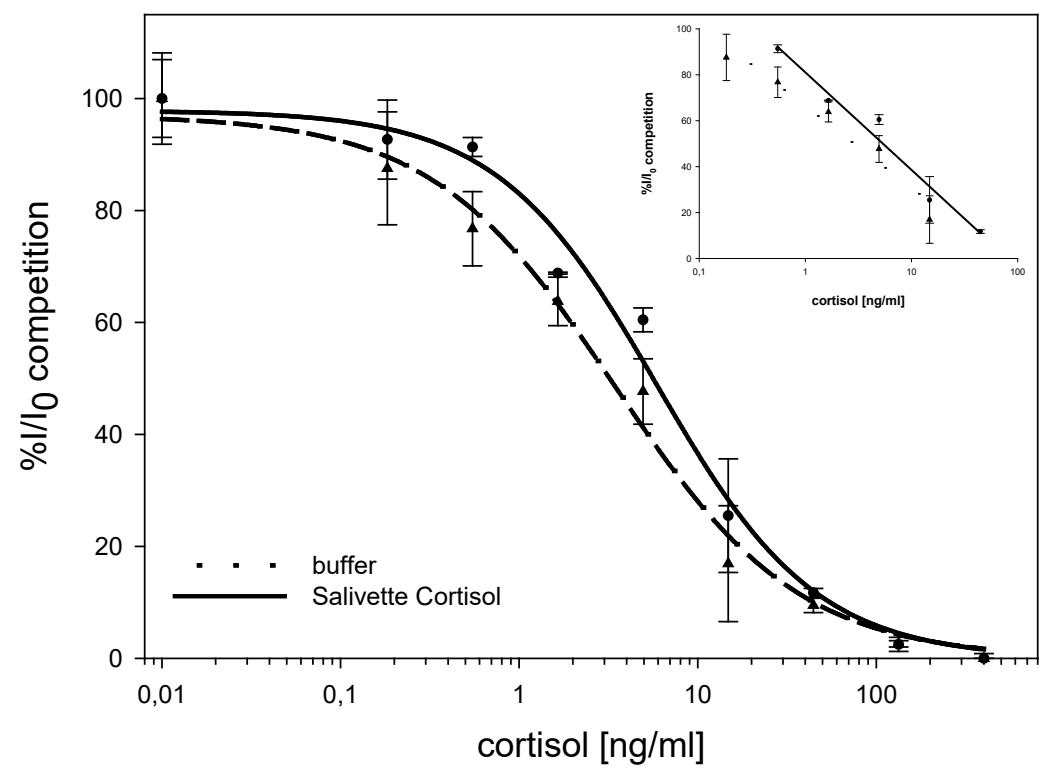


Figure 5. Comparison of calibration curves performed in buffer (dashed line) and saliva collected with Salivette Cortisol (solid line). Endogenous cortisol concentration of saliva was determined with salivary cortisol enzyme immunoassay kit and it was $1.5 \mathrm{ng} / \mathrm{ml}$. 1-NP $(1.5 \mathrm{mg} / \mathrm{ml})$ in DEA solution was measured with SWV $(0.05-0.5 \mathrm{~V}$ range, $4 \mathrm{mV}$ step potential, $25 \mathrm{mV}$ amplitude and $25 \mathrm{~Hz}$ frequency).

Saliva was collected with Salivette Cortisol collection devices and samples were pooled. Pooled saliva samples were spiked with different cortisol concentrations and the amount of endogenous cortisol in samples were determined. Calibration curve performed in saliva showed good reproducibility (RSD 8\%) with only minor decrease in sensitivity with a LOD of $1.7 \mathrm{ng} / \mathrm{ml}$ and WR $0.5-55.1 \mathrm{ng} / \mathrm{ml}$ (figure 5 , table 2). Comparison of linear ranges in buffer and Salivette Cortisol collected saliva showed that sensitivity was higher in saliva than in buffer with slopes -1.20 and $-1.97 \mu \mathrm{A} / \mathrm{ng} / \mathrm{ml}\left(\mathrm{r}^{2}=0.95\right.$ and 0.97$)$, respectively (table 2).

Table 2. Immunosenosr performance parameters in buffer and saliva collected with Salivette Cortisol.

\begin{tabular}{lcc}
\hline Parameter & Buffer & Salivette Cortisol collected saliva \\
\hline Repeatability (\%) & 7 & 6 \\
Reproducibility (\%) & 10 & 8 \\
Sensitivity $\log x(\mu \mathrm{A} / \mathrm{ng} / \mathrm{ml})$ & -1.20 & -1.97 \\
LOD $(\mathrm{ng} / \mathrm{ml})$ & 0.6 & 1.7 \\
$\mathrm{WR}(\mathrm{ng} / \mathrm{ml})$ & $0.2-44.6$ & $0.5-55.1$ \\
$\mathrm{IC}$ & 3.6 & 6.0 \\
\hline
\end{tabular}

It was shown that Salivette Cortisol polyethylene swab could remove interfering compounds in saliva matrix (figure 5) and to evaluate developed immunosensor function a recovery study was conducted by spiking saliva samples with known cortisol concentrations. With four spiked concentrations an excellent recovery was found with the amount of detected cortisol varying from 92 to $114 \%$ (table 3 ).

Table 3. The recovery of cortisol from spiked saliva samples determined using the developed immunosensor $(n=3)$.

$\begin{array}{llll}\text { Cortisol added (ng/ml) } & \text { Cortisol detected (ng/ml) } & \text { \%RSD } & \text { Recovery (\%) }\end{array}$




\begin{tabular}{lccc}
\hline 2.5 & 2.6 & 9 & 104 \\
5.0 & 4.6 & 12 & 92 \\
12.5 & 12.4 & 13 & 99 \\
24.5 & 28.0 & 3 & 114 \\
\hline
\end{tabular}

\subsection{Comparison of immunosensor and UHPLC-MS/MS}

In order to compare developed immunosensor performance to another method 14 spiked saliva samples were analysed with immunosensor and UHPLC-MS/MS method. Saliva samples were collected with Salivette Cortisol and spiked with different amounts of cortisol. Results obtained showed a strong correlation with correlation factor 0.90 between methods and confirmed developed immunosensor usability for salivary cortisol samples measurement (figure 6).

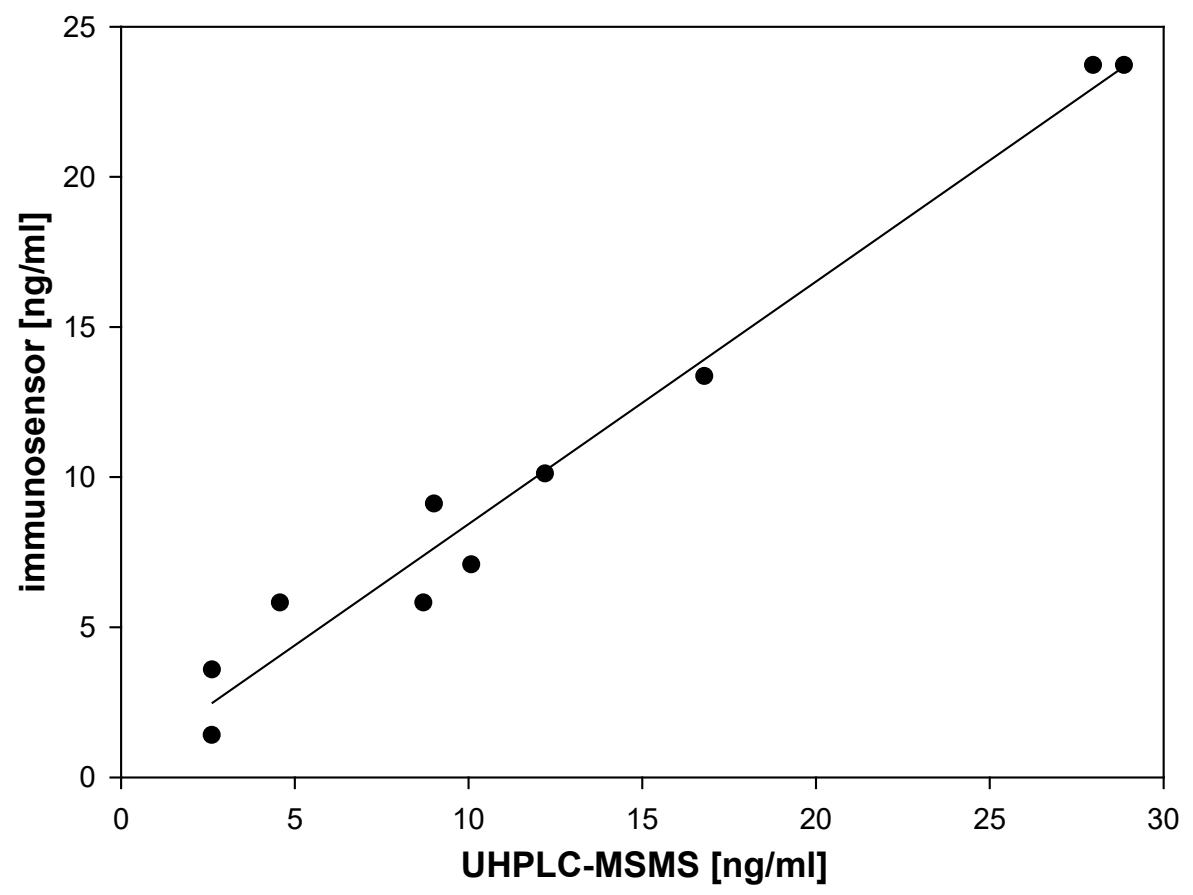

Figure 6. Correlation of cortisol concentrations in spiked saliva samples between electrochemical immunosensor and UHPLC-MS/MS methods.

\section{Conclusions}


A sensitive electrochemical direct competitive immunosensor for measuring cortisol concentration in saliva was developed using a house-made cortisol-AP conjugate. Based on a disposable carbon screen-printed electrodes, the working range of the immunosensor extended from $0.2 \mathrm{ng} / \mathrm{ml}$ to $44.6 \mathrm{ng} / \mathrm{ml}$ and had a detection limit of $0.6 \mathrm{ng} / \mathrm{ml}$ and $1.7 \mathrm{ng} / \mathrm{ml}$ in buffer and saliva, respectively. Hence, allowing the immunosensor to measure human salivary cortisol within the desired physiological range with high reproducibility. Within this study, several saliva sample pretreatments were performed in order to reduce sample matrix interferences affecting the electrochemical response within the immunosensor protocol. Saliva pretreatment for removing interfering matrix components by filtering, diluting or by using chemical degradation of mucin or cotton Salivette collection device proved to be ineffectual and did not eliminate the matrix interference sufficiently as shown by the primary antibody not being able to bind with the free salivary cortisol in the sample. Conversely, saliva samples collected using polyethylene Salivette Cortisol swab showed better suppression of the saliva matrix. The immunosensor showed excellent recovery and saliva samples collected with Salivette Cortisol and spiked with known cortisol concentrations were analysed with the developed immunosensor and with UHPLC-MS/MS method. Results obtained showed strong correlation between both methods. This work demonstrated that a sensitive electrochemical immunosensor capable to quantify physiological cortisol concentrations in whole saliva sample with simple pretreatment was realised and there is a good possibility in the future for integrating it in to a POC device for stress monitoring.

\section{Acknowledgements}

The authors acknowledge the financial support from the Kainuun ELY-keskus and Kainuun maakuntakuntayhtymä through European Regional Development Fund (ERDF) and European Social Fund (ESF). 


\section{REFERENCES}

Aardal-Eriksson, E., Eriksson, T.E., Holm, A-C., Lundin, T., 1999. Salivary cortisol and serum prolactin in relation to stress rating scales in a group of rescue workers. Biological Psychiatry 46, 850-855.

Anfossi, L., Tozzi, C., Giovannoli, C., Baggiani, C., Giraudi, G., 2002. Development of a non-competitive immunoassay for cortisol and its application to the analysis of saliva. Analytica Chimica Acta 468, 315321.

de Almeida, P.D.V., Grégio, A.M.T., Machado, M.Â.N., de Lima, A.A.S., Azevedo, L.R., 2008. Saliva composition and functions: A comprehensive review. The Journal of Contemporary Dental Practice 9, 7280 .

Arafah, B.M., Nishiyama, F.J., Tlaygeh, H., Hejal, R., 2007. Measurement of salivary cortisol concentration in the assessment of adrenal function in critically ill subjects: A surrogate marker of the circulating free cortisol. The Journal of Clinical Endocrinology and Metabolism 92, 2965-1971.

Arya, S.K., Chornokur, G, Venogopal, M., Bhansali, S. (2010) Dithiobis(succinimidyl propionate) modified gold microarray electrode based electrochemical immunosensor for ultrasensitive detection of cortisol. Biosensors and Bioelectronics 25, 2296-2301.

Arya, S.K., Dey, A., Bhansali, S. (2011) Polyaniline protected gold nanoparticles based mediator and label free electrochemical cortisol biosensor. Biosensors and Bioelectronics 28, 166-173.

Gafni, R.I., Papanicolaou, D.A., Nieman, L.K., 2000. Nighttime salivary cortisol measurement as a simple, noninvasive, outpatient screening test for Cushing's syndrome in children and adolescents. The Journal of Pediatrics 137, 30-35.

Gatti, R., Antonelli, G., Prearo, M., Spinella, P., Cappelin, E., De Palo, E.F., 2009. Cortisol assays and diagnostic laboratory procedures in human biological fluids. Clinical Biochemistry 42, 1205-1217.

Gehring, A., Brewster, J.D., Irwin, P.L., Tu, S-I., Van Houten L.J. 1999. 1-Naphthyl phosphate as an enzymatic substrate for enzyme-linked immunomagnetic electrochemistry. Journal of Electroanalytical Chemistry 469 (1999) 27-33.

Gozansky, W.S., Lynn, J.S., Laudenslager, M.L., Kohrt, W.M., 2005. Salivary cortisol determined by enzyme immunoassay is preferable to serum total cortisol for assessment of dynamic hypothalamicpituitary-adrenal axis activity. Clinical Endocrinology 63, 336-341.

Gröschl, M., Köhler, H., Topf, H.G., Ruoorecht, T., Rauh, M., 2008. Evlauation of saliva collection devices for the analysis of steroids, peptides and therapeutic drugs. Journal of Pharmaceutical and Biomedical Analysis 47, 478-486.

Gröschl, M., Rauh, M., 2006. Influence of commercial collection devices for saliva on the reliability of salivary steroid analysis. Steroids 71, 1097-1100. 
Holsboer, F., Ising, M., 2010. Stress hormone regulation: Biological role and translation into therapy. Annual Review of Psychology 61, 81-109.

Jensen, M. A., Hansen, Å. M., Nørgaard, A. W., 2011. Development and Evaluation of Liquid Chromatography Tandem Mass Spectrometry Method for Simultaneous Determination of Salivary Melatonin, Cortisol and Testosterone. Journal of Chromatography B, 879: 2527-2532.

Jönsson, B.A.G., Malmberg, B., Amilon, Å., Garde, A.H., Ørbæk, P., 2003. Determination of cortisol in human saliva using liquid chromatography-electrospray tandem mass spectrometry. Journal of Chromatography B 784, 63-68.

Kaushik, A., Vasudev, A., Arya, S.K., Pasha, S.K., Bhansail, S. 2014. Recent advances in cortisol sensing technology for point of care application. Biosensors and Bioelectronics 53, 499-512.

Krotzky, A.J. \& Zeeh, B. (1995) Immunoassays for residue analysis of agrochemicals: proposed guidelines for precision, standardization and quality control. IUPAC, Pure \& Applied Chemistry 67, 2065 - 2088.

Kumar, A., Aravamudhan, S., Gordic, M., Bhansali, S., Mohapatra, S.S. (2007) Ultrasensitive detection of cortisol with enzyme fragment complementation technology using functionalized nanowire. Biosensors and bioelectronics 22, 2138-2144.

Lee, A-C., Liu, G., Heng, C-K., Tan, S-N., Lim, T-M., Lin, Y. 2008. Sensitive electrochemical detection of horseradish peroxidase at disposable screen-printed carbon electrode. Electroanalysis 20, 2040-2046.

Lewis, J.G., 2006. Steroid analysis in saliva: An overview. The Clinical Biochemist Reviews 27, 139-146.

Lin, Y-Y., Liu, G., W, C.M., L, Y., 2008. Bioelectrochemical immunoassay of polychlorinated biphenyl. Analytica Chimica Acta 612, 23-28.

Liu, G., Timchalk, C., Lin, Y. 2006. Bioelectrochemical magnetic immunosensing of trichloropyridinol: A potential insecticide biomarker. Electroanalysis 18, 1605-1613.

Long, J.D. \& Winefordner, J.D. 1983 Limit of detection: A closer look at the IUPAC definition. Analytical chemistry $55,712-724$.

McNaught, A.D. \& Wilkinson, A. (Eds.) 2014 IUPAC. Compendium of Chemical Terminology, version 2.3.3 (the "Gold Book"), Blackwell Scientific Publications, Oxford, p. 839.

Miller, R., Plessow, F., Rauh, M., Gröschl, M., Kirschbaum, C., 2013. Comparison of salivary cortisol as measured by different immunoassays and tandem mass spectrometry. Psychoneuroendocrinology 38, 5057.

Mitchell, J.S., Lowe, T.E., 2009. Matrix effect on an antigen immobilized format for competitive enzyme immunoassay of salivary testosterone. Journal of Immunological methods 349, 61-66.

Morineau, G., Boudi, A., Barka, A., Gourmelen, M., Degeilh, F., Hardy, N., Al-Halnak, A., Soliman, H., Gosling, J.P., Julien, R., Brerault, J-L., Boudou, P., Aubert, P., Villette, J-M., Pruna, A., Galons, H., Fiet, 
J., 1997. Radioimmunoassay of cortisone in serum, urine, and saliva to assess the status of the cortisolcortisone shuttle. Clinical Chemistry 43, 1397-1407.

Nomura, S., Fujitaka, M., Sakura, N., Ueda, K., 1997. Circadian rhythms in plasma cortisone and cortisol and the cortisone/cortisol ratio. Clinica Chimica Acta 266, 83-91.

Nozaki, O., 2001. Steroid analysis for medical diagnosis. Journal of Chromatography A 935, 267-278.

Pemberton, R.M., Mottram, T.T., Hart, J.P., 2005. Development of a screen-printed carbon electrochemical immunosensor for picomolar concentrations of estradiol in human serum extracts. Journal of Biochemical and Biophysical Methods 63, 201-212.

Perogamvros, I., Keevil, B.G., Ray, D.W., Trainer, P.J., 2010. Salivary cortisol is a potential Biomarker for serum free cortisol. The Journal of Clinical Endocrinology and Metabolism 95, 4951-4958.

Perogamvros, I., Owen, L.J., Newell-Price, J., Ray, D.W., Trainer, P.J., Keevil, B.G., 2009. Simultaneous measurement of cortisol and cortisone in human saliva using liquid chromatography-tandem mass spectrometry: Application in basal and stimulated conditions. Journal of Chromatography B 877, 37713775.

Pillai, K., Akhter, J., Chua, T.C., Morris, D.L., 2012. Potential mucolytic agents for mucinous ascites from pseudomyxoma peritonei. Invest New Drugs 30, 2080-2086.

Preechaworapun, A., Dai, Z., Xiang, Y., Chailapakul, O., Wang, J. 2008. Investigation of the enzyme hydrolysis products of the substrates of alkaline phosphatase in electrochemical immunosensing. Talanta $76,424-431$.

Pruessner, J.C., Hellhammer, D.H., Kirschbaum, C., 1999. Burnout, perceived stress, and cortisol responses to awakening. Psychosomatic Medicine 61, 197-204.

Rao, V.K., Rai, G.P., Agarwal, G.S., Suresh, S., 2007. Amperometric immunosensor for detection of antibodies of Salmonella typhi in patient serum. Analytica Chimica Acta 531, 173-177.

Robertson, W.v.B., Ropes, M.W., Bauer, W., 1941. The degradation of mucins and polysaccharides by ascorbic acid and hydrogen peroxide. Biochemical Journal 35, 903-908.

Rodbard, D. (1974) Statistical quality control and routine data processing for radioimmunoassay and immunoradiometric asssays. Analytical Chemistry 20, 1255-1270.

Sesay, A.M., Micheli, L., Tervo, P., Palleschi, G., Virtanen, V., 2013. Development of a competitive immunoassay for the determination of cortisol in human saliva. Analytical Biochemistry 434, 308-314.

Strazdins, L., Meyerkort, S., Brent, V., D’Souza, R.M., Broom, D.H., Kyd, J.M., 2005. Impact of saliva collection methods on SIgA and cortisol assays and acceptability to participants. Journal of Immunological Methods 307, 167-171. 
Strickland, P., Morriss, R., Wearden, A., Deakin, B., 1998. A comparison of salivary cortisol in chronic fatigue syndrome, community depression and healthy controls. Journal of Affective Disorders 47, 191-194.

Sun, K., Ramgir, N., Bhansali, S. (2008) An immunoelectrochemical sensor for salivary cortisol measurement. Sensors and Actuators B 133, 533-537.

Tlili, C., Myung, N.V., Shetty, V., Mulchandani, A. (2011) Label-free, chamiresistor immunosensor for stress biomarker cortisol in saliva. Biosensors and Bioelectronics, 26:4382-4386.

Vasudev, A., Kaushik, A., Tomizawa, Y., Norena, N., Bhansali, S. (2013) An LTCC-based microfluidistic system for label-free, electrochemical detection of cortisol. Sensors and Actuators B 182, 139-146.

Venogopal, M., Arya, S.K., Chornokur, G., Bhansali, S. (2011) A realtime and continuous assessment of cortisol in ISF using electrochemical impedance spectroscopy. Sensors and Actuators A 172, 154-160.

Vining, R.F., McGinley, R.A., Symons, R.G., 1983. Hormones in saliva: Mode of entry and consequent implications for clinical interpretation. Clinical Chemistry 29, 1752-1756.

Warwick, M.J., 2005. Standardisation of immunoassays. In Immunoassay: A Practical Guide. Ed. Law, B. Taylor \& Francis Ltd, London, UK. p. 157-178.

Yamaguchi, M., Matsuda, Y., Sasaki, S., Sasaki, M., Kadoma, Y., Imai, Y., Niwa, D., Shetty, V., 2013. Immunosensor with fluid control mechanism for salivary cortisol analysis. Biosensors and Bioelectronics 41, 186-191. 